\title{
The influence of hardware impairment on the system performance of two-way relaying network
}

\author{
Le Anh Vu ${ }^{1}$, Minh Tran ${ }^{2}$, Van-Duc Phan ${ }^{3}$, Hoang-Nam Nguyen ${ }^{4}$, Thanh-Long Nguyen ${ }^{5}$ \\ ${ }^{1,2}$ Optoelectronics Research Group, Faculty of Electrical and Electronics Engineering, \\ Ton Duc Thang University, Vietnam \\ ${ }^{3}$ Center of Excellence for Automation and Precision Mechanical Engineering, \\ Nguyen Tat Thanh University, Vietnam \\ ${ }^{4}$ Modeling Evolutionary Algorithms Simulation and Artificial Intelligence, \\ Faculty of Electrical and Electronics Engineering, Ho Chi Minh City, Vietnam \\ ${ }^{5}$ Center for Information Technology, Ho Chi Minh City University of Food Industry, Vietnam
}

\begin{tabular}{ll} 
Article Info & ABSTRACT \\
\cline { 1 - 2 } Article history: & $\begin{array}{l}\text { In this research, the system performance of the half-duplex two-way relay } \\
\text { network under hardware impairment condition is presented and investigated. }\end{array}$ \\
$\begin{array}{l}\text { Received Feb 27, 2019 } \\
\text { Revised Apr 5, 2019 }\end{array}$ & $\begin{array}{l}\text { In this system model, the time switching protocol and amplify-and-forward } \\
\text { (AF)-based are considered. First, of all, the analytical expressions of } \\
\text { the outage probability, and achievable throughput with the exact closed form } \\
\text { and asymptotic form were proposed and derived. Furthermore, the research } \\
\text { results are obtained and convinced by Monte-Carlo calculation. } \\
\text { Keywords: }\end{array}$ \\
$\begin{array}{l}\text { The numerical results demonstrated and convinced the analytical and the } \\
\text { simulation results are agreed for all possible system parameters. }\end{array}$
\end{tabular}

Hardware impairment

Monte Carlo simulations

Outage probability

Throughput

Copyright $@ 2019$ Institute of Advanced Engineering and Science. All rights reserved.

\section{Corresponding Author:}

Van-Duc Phan

Center of Excellence for Automation and Precision Mechanical Engineering,

Nguyen Tat Thanh University,

Ho Chi Minh City, Vietnam.

Email:pvduc@ntt.edu.vn

Hoang-Nam Nguyen,

Modeling Evolutionary Algorithms Simulation and Artificial Intelligence,

Faculty of Electrical \& Electronics Engineering,

Ton Duc Thang University,

Ho Chi Minh City, Vietnam.

Email: nguyenhoangnam@tdtu.edu.vn

\section{INTRODUCTION}

Generally, one-way relaying network with one-way information transmission is the typical way to improve the system performance regarding coverage, throughput, and reliability [1-3]. Regularly, bidirectional information transmission between source and destination nodes via a relay in the relaying network requires four channels. On another hand, this information can be exchanged efficiently by two channel over two-way relaying networks. In such this relay network, the bidirectional informationtransmission are proposed. From that point of view, the spectral efficiency of two-way relay network is twice that of the one-way relay network. According to the massive improvements, the two-way relaying network has been considered as a next-generation wireless communication network [4-7]. 
In the last decades, many works focus on the system performance of the two-way relay network. In common, the outage probability for the two-way relaying network has been investigated in details in $[8,9]$ with the Rayleigh and in $[10,11]$ with the Nakagami-m fading channels. Moreover, the outage performance of the two-way relaying network with outdated channel state information is proposed in [12] over a Rayleigh fading channel and is extended to multi-user, multi-relay in [13, 14]. As far as authors' knowledge, there are not many works to concentrate on the system performance analysis of the two-way amplify-and-forward (AF)-based relay network over the Rician fading channels in the influence of the hardware impairment yet. It is the remaining gap could be filled in by this research.

In this research, the system performance analysis of the half-duplex two-way relaying network under hardware impairment over the Rician fading channels is proposed in details. For this purpose, the exact closed-form and the asymptotic expressions of the achievable throughput, outage probability of the model system were introduced and derived. Furthermore, the analytical results could be demonstrated and convinced by Monte-Carlo simulation with helping Mat Lab software. The research results show that the analytical and simulated results are agreed for all proposed system parameters. The research results provided the practical solution for the progress of the WPCN. Here, we can recommend the main contributions of the paper as follows:

- The system model of the half-duplex two-way relay network under hardware impairment over the Rician fading channels is proposed.

- The exact closed-form and asymptotic expressions of the throughput, and the outage probability proposed system is derived.

- The influence of hardware impairment on system performance is demonstrated entirely.

The remaining of this paper is proposed as follows. Sections II presents the proposed system model. Sections III introduces and demonstrates the analytical expressions of the throughput and outage probability of the proposed system. Section IV gives numerical results and some discussions. Finally, Section V concludes the paper.

\section{SYSTEM MODEL AND SYSTEM PERFORMANCE ANALYSIS}

Figure 1 presents the system model of the half-duplex two-way relay network under hardware impairment. In Figure 1, the information is transferred between the nodes S1 and S2, through an intermediate relay $(\mathrm{R})$. The information processing at the relay is proposed in Figure 2 with $\mathrm{T}$ is the block time. In this proposed system model, the nodes S1, S2 transfer information signal to the relay node R in the first interval time $T / 2$, then the relay $\mathrm{R}$ transfers the information signal back to the nodes $\mathrm{S} 1, \mathrm{~S} 2$ in the remaining interval time $T / 2[15,16]$.

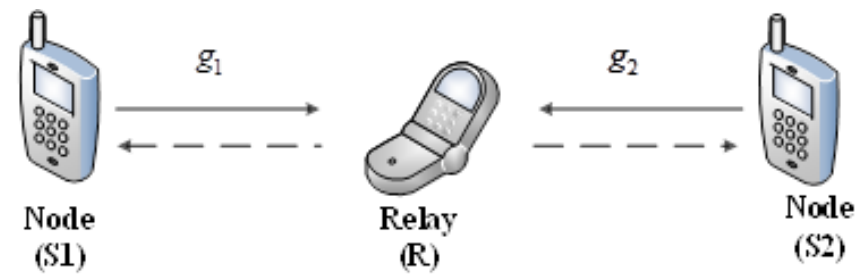

Figure 1. System model

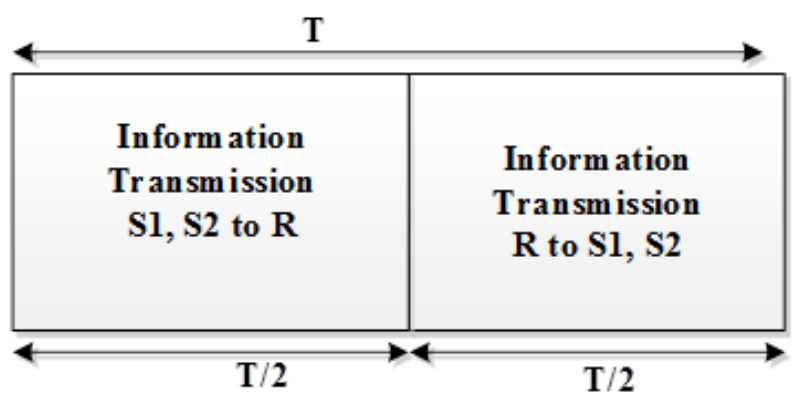

Figure 2. The energy harvesting and information processing 
In this section, a three-node relaying network is considered. In this model, each node operates in half-duplex mode and has a single antenna. In this network, two nodes S1 and S2 have no direct connection and communicate with each other via the help of relay $R$ over Rician fading channels. In the first interval time $T / 2$, both nodes $S 1$ and $S 2$ simultaneously transfer their signal to relay node $R$. The received signal at the relay node $\mathrm{R}$ can be calculated as the following:

$$
y_{r}=\sum_{i=1}^{2} g_{i}\left(s_{i}+\eta_{i}\right)+n_{r}, \mathrm{i}=1,2
$$

where $\mathrm{E}\left[\left|s_{i}\right|^{2}\right]=P_{i}, \mathrm{~S}_{\mathrm{i}}$ : transmit signal at node $\mathrm{I}, \mathrm{P}_{\mathrm{i}}$ : transmit power at the node $\mathrm{I}, \eta_{i}$ denotes the distortion noise with zero mean and variance $\kappa_{i}^{2} P_{i}, \kappa_{i}$ is the aggregate level of impairments of the channel, and $n_{r}$ is the additive white Gaussian noise (AWGN) at $R$ with zero mean and variance $\sigma_{r}^{2}$.

The received signal of node $S_{i}$ from $R$ can be given by:

$$
y_{i}=g_{i}\left(r+\eta_{r}\right)+n_{i}, \mathrm{i}=1,2
$$

where $\mathrm{r}$ is the transmit signal at $\mathrm{R}, \eta_{r}$ denotes the distortion noise with zero mean and variance $k_{r}^{2} P_{r}, n_{i}$ is the additive white Gaussian noise (AWGN) at $S_{\mathrm{i}}$ with zero mean and variance $\sigma_{i}^{2}$, and $\kappa_{r}$ is the aggregate level of impairments of the channel.

In this analysis amplify factor can be formulated as the following:

$$
\beta=\frac{r}{y_{r}}=\sqrt{\frac{P_{r}}{\varphi_{1} P_{1}+\varphi_{1} P_{1} \kappa_{1}^{2}+\varphi_{2} P_{2}+\varphi_{2} P_{2} \kappa_{2}^{2}+\sigma_{r}^{2}}}
$$

Where $\varphi_{i}=\left|g_{i}\right|^{2}$ denotes the channel gain between nodes $\mathrm{S}_{\mathrm{i}}$ and relay $\mathrm{R}$.

\subsection{The exact analysis}

In this proposed system, because of the symmetry of $S 1$ and $S 2$, we first provide the expression of the $\mathrm{y}_{1}$ :

$$
y_{1}=g_{1}\left(r+\eta_{r}\right)+n_{1}=g_{1}\left(\beta y_{r}+\eta_{r}\right)+n_{1}
$$

By substituting (1) and (2) into (4), we obtain:

$$
y_{1}=\beta\left|g_{1}\right|^{2} s_{1}+\beta\left|g_{1}\right|^{2} \eta_{1}+\beta\left|g_{1}\right|\left|g_{2}\right| s_{2}+\beta\left|g_{1}\right|\left|g_{2}\right| \eta_{2}+\beta\left|g_{1}\right| n_{r}+\left|g_{1}\right| \eta_{r}+n_{1}
$$

$S_{1}$ wants to extract $s_{2}$ from $y 1$. Since it knows its transmitted symbol $s 1$, it can correctly eliminate the similar self-interference term $\beta\left|g_{1}\right|^{2} s_{1}$.

From (5), we can rewrite:

$$
y_{1}=\underbrace{\beta\left|g_{1}\right|\left|g_{2}\right| s_{2}}_{\text {signal }}+\underbrace{\beta\left|g_{1}\right|^{2} \eta_{1}+\beta\left|g_{1}\right|\left|g_{2}\right| \eta_{2}+\beta\left|g_{1}\right| n_{r}+\left|g_{1}\right| \eta_{r}+n_{1}}_{\text {noise }}
$$

Therefore, the end to end signal to noise ratio (SNR) at $S_{1}$ for detection of the symbol $\mathrm{y}_{1}$ is given by [1]:

$$
\gamma_{1}=\frac{\mathrm{E}\left\{\mid \text { signal }\left.\right|^{2}\right\}}{\mathrm{E}\left\{\mid \text { noise }\left.\right|^{2}\right\}}=\frac{\beta^{2}\left|g_{1}\right|^{2}\left|g_{2}\right|^{2} P_{2}}{\beta^{2}\left|g_{1}\right|^{4} P_{1} \kappa_{1}^{2}+\beta^{2}\left|g_{1}\right|^{2}\left|g_{2}\right|^{2} P_{2} \kappa_{2}^{2}+\beta^{2}\left|g_{1}\right|^{2} \sigma_{r}^{2}+\left|g_{1}\right|^{2} P_{r} \kappa_{r}^{2}+\sigma_{1}^{2}}
$$

After doing some algebra, we obtain the final expression for $\mathrm{y}_{1}$ : 


$$
\gamma_{1}=\frac{\varphi_{1} \varphi_{2}}{\varphi_{1} \varphi_{2} A_{1}+\varphi_{1}^{2} B_{1}+\varphi_{1} C_{1}+\varphi_{2} D_{1}+E_{1}}
$$

where we denote:

$$
A_{1}=\kappa_{2}^{2}+\kappa_{r}^{2}\left(1+\kappa_{2}^{2}\right), B_{1}=\left\{\kappa_{1}^{2}+\kappa_{r}^{2}\left(1+\kappa_{1}^{2}\right)\right\} \frac{P_{1}}{P_{2}} \begin{aligned}
& C_{1}=\frac{\sigma_{r}^{2}}{P_{2}}+\left(1+\kappa_{1}^{2}\right) \frac{P_{1} \sigma_{1}^{2}}{P_{r} P_{2}}+\kappa_{r}^{2} \frac{\sigma_{r}^{2}}{P_{2}} \\
& D_{1}=\left(1+\kappa_{2}^{2}\right) \frac{\sigma_{1}^{2}}{P_{r}}, E_{1}=\frac{\sigma_{r}^{2} \sigma_{1}^{2}}{P_{r} P_{2}}
\end{aligned}
$$

By the same way, the end to end signal to noise ratio (SNR) of $\mathrm{y}_{2}$ is also given by the following:

$$
\gamma_{2}=\frac{\varphi_{1} \varphi_{2}}{\varphi_{1} \varphi_{2} A_{2}+\varphi_{2}^{2} B_{2}+\varphi_{2} C_{2}+\varphi_{1} D_{2}+E_{2}}
$$

where we denote:

$$
\begin{aligned}
& A_{2}=\kappa_{1}^{2}+\kappa_{r}^{2}\left(1+\kappa_{1}^{2}\right), B_{2}=\left\{\kappa_{2}^{2}+\kappa_{r}^{2}\left(1+\kappa_{2}^{2}\right)\right\} \frac{P_{2}}{P_{1}}, \\
& C_{2}=\frac{\sigma_{r}^{2}}{P_{1}}+\left(1+\kappa_{2}^{2}\right) \frac{P_{2} \sigma_{2}^{2}}{P_{r} P_{1}}+\kappa_{r}^{2} \frac{\sigma_{r}^{2}}{P_{1}}, D_{2}=\left(1+\kappa_{1}^{2}\right) \frac{\sigma_{2}^{2}}{P_{r}}, E_{2}=\frac{\sigma_{r}^{2} \sigma_{2}^{2}}{P_{r} P_{1}}
\end{aligned}
$$

Theorem 1. The exact closed-form expression of the outage probability $P_{\text {out_l }}$ and $P_{\text {out_2 }}$ at the source nodes S1, S2 of the proposed system could be calculated by the following:

$$
\begin{aligned}
& P_{\text {out }-1}=1-2 a^{2} e^{-b\left(\frac{B_{1} \gamma_{t h} D_{1}}{\vartheta_{1}{ }^{2}}+\theta_{1}+\frac{\gamma_{t h D_{1}}}{\vartheta_{1}}\right)} \\
& \sum_{l=0}^{\infty} \sum_{k=0}^{\infty} \sum_{v=0}^{k} \sum_{m=0}^{l} \sum_{n=0}^{m} \sum_{p=0}^{m-n} \frac{K^{l+k} b^{m+k-1} \gamma_{t h}^{m+v} B_{1}^{m-n-p} \xi_{1}^{n} D_{1}^{v}}{l ! n ! v ! p ! k !(m-n-p) !(k-v) ! \vartheta_{1}^{p-m-v}} \\
& \times\left(\frac{B_{1} \gamma_{t h} D_{1}}{\vartheta_{1}^{2}}+\theta_{1}\right)^{p} \times\left(\frac{\xi_{1}}{B_{1}+\vartheta_{1}}\right)^{\frac{m-2 n-p+k-v+1}{2}} \times K_{m-2 n-p+k-v+1}\left(2 \frac{b}{\vartheta_{1}} \sqrt{\left(B_{1}+\vartheta_{1}\right) \xi_{1}}\right) \\
& P_{\text {out }-2}=1-2 a^{2} e^{-b\left(\frac{B_{2} \gamma_{h t} D_{2}}{\vartheta_{2}{ }^{2}}+\theta_{2}+\frac{\gamma_{t h D_{2}}}{\vartheta_{2}}\right)} \\
& \sum_{l=0}^{\infty} \sum_{k=0}^{\infty} \sum_{v=0}^{k} \sum_{m=0}^{l} \sum_{n=0}^{m} \sum_{p=0}^{m-n} \frac{K^{l+k} b^{m+k-1} \gamma_{t h}^{m+v} B_{2}^{m-n-p} \xi_{2}{ }^{n} D_{2}{ }^{v}}{l ! n ! v ! p ! k !(m-n-p) !(k-v) ! \vartheta_{2}{ }^{p-m-v}} \\
& \times\left(\frac{B_{2} \gamma_{t h} D_{2}}{\vartheta_{2}{ }^{2}}+\theta_{2}\right)^{p} \times\left(\frac{\xi_{2}}{B_{2}+\vartheta_{2}}\right)^{\frac{m-2 n-p+k-v+1}{2}} \times K_{m-2 n-p+k-v+1}\left(2 \frac{b}{\vartheta_{2}} \sqrt{\left(B_{2}+\vartheta_{2}\right) \xi_{2}}\right)
\end{aligned}
$$

\section{Proof:}

The probability density function (PDF) of a random variable (RV) $\varphi_{i}$ where $\mathrm{i}=1,2$ can be calculated as in [17].

$$
f_{\varphi_{i}}(x)=\frac{(K+1) e^{-K}}{\lambda_{i}} e^{-\frac{(K+1) x}{\lambda_{i}}} I_{0}\left(2 \sqrt{\frac{K(K+1) x}{\lambda_{i}}}\right)
$$

In which $\lambda_{i}$ is the mean value of $\operatorname{RV} \varphi_{i}$ which $\mathrm{i}=1,2$ respectively. Moreover, $\mathrm{K}$ is the Rician $\mathrm{K}$-factor defined as the ratio of the power of the line-of-sight (LOS) component to the separate components and $I_{0}(\bullet)$ is the zero-th order modified Bessel function of the first kind.

The (A1) can be rewritten as follow: 


$$
f_{\varphi_{i}}(x)=a \sum_{l=0}^{\infty} \frac{(b K)^{l}}{(l !)^{2}} x^{l} e^{-b x}
$$

where we denote $a=\frac{(K+1) e^{-K}}{\lambda_{i}}, b=\frac{K+1}{\lambda_{i}}$ and $I_{0}(x)=\sum_{l=0}^{\infty} \frac{x^{2 l}}{2^{2 l}(l !)^{2}}[18]$.

The cumulative density function (CDF) of $\mathrm{RV} \varphi_{i}$ where $\mathrm{i}=1,2$ can be computed as in [19] with $\lambda_{1}=\lambda_{2}$ :

$$
F_{\varphi_{i}}(\varsigma)=\int_{0}^{\varsigma} f_{\varphi_{i}}(x) d x=1-\frac{a}{b} \sum_{l=0}^{\infty} \sum_{m=0}^{l} \frac{K^{l} b^{m}}{l ! m !} \varsigma^{m} e^{-b \varsigma}
$$

We have the outage probability at the source S1:

$$
\begin{aligned}
& P_{\text {out }-1}=F_{\gamma_{1}}\left(\gamma_{t h}\right)=\operatorname{Pr}\left(\gamma_{1}<\gamma_{t h}\right) \\
& =\operatorname{Pr}\left(\frac{\varphi_{1} \varphi_{2}}{\varphi_{1} \varphi_{2} A_{1}+\varphi_{1}^{2} B_{1}+\varphi_{1} C_{1}+\varphi_{2} D_{1}+E_{1}}<\gamma_{t h}\right)
\end{aligned}
$$

where $\gamma_{t h}=2^{2 R}-1$ and $\mathrm{R}$ is the source rate.

From (A4) we have:

$$
\begin{aligned}
& \operatorname{Pr}\left(\frac{\varphi_{1} \varphi_{2}}{\varphi_{1} \varphi_{2} A_{1}+\varphi_{1}^{2} B_{1}+\varphi_{1} C_{1}+\varphi_{2} D_{1}+E_{1}}<\gamma_{t h}\right) \\
& =\operatorname{Pr}\left\{\varphi_{2}\left(\varphi_{1}-\gamma_{t h} \varphi_{1} A_{1}-\gamma_{t h} D_{1}\right)<\gamma_{t h} \varphi_{1}^{2} B_{1}+\gamma_{t h} \varphi_{1} C_{1}+\gamma_{t h} E_{1}\right\} \\
& =\left\{\begin{array}{ll}
\operatorname{Pr}\left\{\varphi_{2}<\frac{\gamma_{t h} \varphi_{1}^{2} B_{1}+\gamma_{t h} \varphi_{1} C_{1}+\gamma_{t h} E_{1}}{\varphi_{1}-\gamma_{t h} \varphi_{1} A_{1}-\gamma_{t h} D_{1}},\right. & \left.\varphi_{1} \geq \frac{\gamma_{t h} D_{1}}{1-\gamma_{t h} A_{1}}\right\} \\
1, & , \varphi_{1}<\frac{\gamma_{t h} D_{1}}{1-\gamma_{t h} A_{1}}
\end{array}\right\}
\end{aligned}
$$

In this analysis, we assume that $1-\gamma_{t h} A_{1}$ is positive because if it is negative then the probability is always equal 1. Furthermore:

$$
\begin{aligned}
& P_{\text {out }-1}=\int_{\frac{\gamma_{h t} D_{1}}{1-\gamma_{h h} A_{1}}}^{\infty} F_{\varphi_{2}}\left(\frac{\gamma_{t h} \varphi_{1}^{2} B_{1}+\gamma_{t h} \varphi_{1} C_{1}+\gamma_{t h} E_{1}}{\varphi_{1}-\gamma_{t h} \varphi_{1} A_{1}-\gamma_{t h} D_{1}}\right) f_{\varphi_{1}} d \varphi_{1} \\
& +\int_{0}^{\frac{\gamma_{1} D_{1}}{1-\gamma_{1} A_{1}}} f_{\varphi_{1}} d \varphi_{1}
\end{aligned}
$$

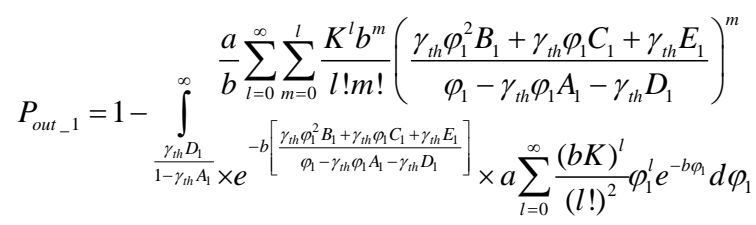

Here, we consider:

$$
\begin{aligned}
& I=\frac{\varphi_{1}^{2} B_{1}+\varphi_{1} C_{1}+E_{1}}{\varphi_{1}-\gamma_{t h} \varphi_{1} A_{1}-\gamma_{t h} D_{1}}=\frac{\varphi_{1}^{2} B_{1}+\varphi_{1} C_{1}+E_{1}}{\vartheta_{1} \varphi_{1}-\gamma_{t h} D_{1}}=\frac{B_{1} \varphi_{1}}{\vartheta_{1}}+\frac{C_{1}}{\vartheta_{1}}+\frac{\gamma_{t h} B_{1} D_{1}}{\vartheta_{1}^{2}} \\
& +\frac{E_{1}+\gamma_{t h} D_{1}\left(\frac{C_{1}}{\vartheta_{1}}+\frac{\gamma_{t h} B_{1} D_{1}}{\vartheta_{1}^{2}}\right)}{\vartheta_{1} \varphi_{1}-\gamma_{t h} D_{1}}=\frac{B_{1} \varphi_{1}}{\vartheta_{1}}+\theta_{1}+\frac{\xi_{1}}{\vartheta_{1} \varphi_{1}-\gamma_{t h} D_{1}}
\end{aligned}
$$


In which we denote $\vartheta_{1}=1-\gamma_{t h} A_{1}, \theta_{1}=\frac{C_{1}}{\vartheta_{1}}+\frac{\gamma_{t h} B_{1} D_{1}}{\vartheta_{1}^{2}}, \xi_{1}=E_{1}+\gamma_{t h} D_{1}\left(\frac{C_{1}}{\vartheta_{1}}+\frac{\gamma_{t h} B_{1} D_{1}}{\vartheta_{1}^{2}}\right)$.

After that, the (A7) can be rewritten as the following:

$$
P_{\text {out_1 }}=1-\int_{\frac{\gamma_{t h} D_{1}}{1-\gamma_{t h} A_{1}} \times e^{-b\left[\frac{B_{1} \varphi_{1}}{\vartheta_{1}}+\theta_{1}+\frac{\xi_{1}}{\vartheta_{1} \varphi_{1}-\gamma_{t h} D_{1}}\right]} \times a \sum_{l=0}^{\infty} \frac{(b K)^{l}}{(l !)^{2}} \varphi_{1}^{l} e^{-b \varphi_{1}} d \varphi_{1} \sum^{l} \frac{K^{l} b^{m} \gamma_{t h}^{m}}{l ! m}\left(\frac{B_{1} \varphi_{1}}{\vartheta_{1}}+\theta_{1}+\frac{\xi}{\vartheta_{1} \varphi_{1}-\gamma_{t h} D_{1}}\right)^{m}}
$$

By changing variable $t=\vartheta_{1} \varphi_{1}-\gamma_{t h} D_{1}$ into the (A9), we have:

$$
\begin{gathered}
P_{\text {out }-1}=1-\int_{0}^{\infty} \frac{a^{2}}{b} \sum_{l=0}^{\infty} \sum_{k=0}^{\infty} \sum_{m=0}^{l} \frac{K^{l+k} b^{m+k} \gamma_{t h}^{m}}{l ! m !(k !)^{2}}\left(\frac{B_{1} t}{\vartheta_{1}^{2}}+\frac{B_{1} \gamma_{t h} D_{1}}{\vartheta_{1}^{2}}+\theta_{1}+\frac{\xi_{1}}{t}\right)^{m} \\
\times e^{-b\left[\frac{B_{1} t}{\vartheta_{1}^{2}}+\frac{B_{1} \gamma_{t h} D_{1}}{\vartheta_{1}^{2}}+\theta_{1}+\frac{\xi_{1}}{t}\right]} \times\left(\frac{t+\gamma_{t h} D_{1}}{\vartheta_{1}}\right)^{k} e^{-b\left(\frac{t+\gamma_{t h} D_{1}}{\vartheta_{1}}\right)} \frac{d t}{\vartheta_{1}}
\end{gathered}
$$

Apply the equation $(x+y)^{m}=\sum_{n=0}^{m}\left(\begin{array}{l}m \\ n\end{array}\right) x^{m-n} y^{n}$ into (A10) we have:

$$
\begin{aligned}
& \begin{aligned}
P_{\text {out } \_1}=1-\int_{0}^{\infty} & \frac{a^{2}}{b} \sum_{l=0}^{\infty} \sum_{k=0}^{\infty} \sum_{m=0}^{l} \frac{K^{l+k} b^{m+k} \gamma_{t h}^{m}}{l ! m !(k !)^{2}} \sum_{n=0}^{m}\left(\begin{array}{l}
m \\
n
\end{array}\right)\left(\frac{B_{1} t}{\vartheta_{1}^{2}}+\frac{B_{1} \gamma_{t h} D_{1}}{\vartheta_{1}^{2}}+\theta_{1}\right)^{m-n}\left(\frac{\xi_{1}}{t}\right)^{n} \\
& e^{-b\left[\frac{B_{1} t}{\vartheta_{1}^{2}}+\frac{B_{1} \gamma_{t h} D_{1}}{\vartheta_{1}^{2}}+\theta_{1}+\frac{\xi_{1}}{t}\right]} \times\left(\frac{t+\gamma_{t h} D_{1}}{\vartheta_{1}}\right)^{k} \times e^{-b\left(\frac{t+\gamma_{t h} D_{1}}{\vartheta_{1}}\right)} \frac{d t}{\vartheta_{1}}
\end{aligned} \\
& \frac{a^{2}}{b} \sum_{l=0}^{\infty} \sum_{k=0}^{\infty} \sum_{m=0}^{l} \frac{K^{l+k} b^{m+k} \gamma_{t h}^{m}}{l ! m !(k !)^{2}} \sum_{n=0}^{m}\left(\begin{array}{c}
m \\
n
\end{array}\right)_{p=0}^{m-n}\left(\begin{array}{c}
m-n \\
p
\end{array}\right)\left(\frac{B_{1} t}{\vartheta_{1}^{2}}\right)^{m-n-p} \\
& P_{\text {out_1 }}=1-\int_{0}^{\infty}\left(\frac{B_{1} \gamma_{t h} D_{1}}{\vartheta_{1}^{2}}+\theta_{1}\right)^{p}\left(\frac{\xi_{1}}{t}\right)^{n} \times e^{-b\left[\frac{B_{1} t}{\vartheta_{1}^{2}}+\frac{B_{1} \gamma_{t h} D_{1}}{\vartheta_{1}^{2}}+\theta_{1}+\frac{\xi_{1}}{t}\right]} \\
& \times \sum_{v=0}^{k}\left(\begin{array}{l}
k \\
v
\end{array}\right)\left(\frac{t}{\vartheta_{1}}\right)^{k-v}\left(\frac{\gamma_{t h} D_{1}}{\vartheta_{1}}\right)^{v} \times e^{-b\left(\frac{t+\gamma_{t h} D_{1}}{\vartheta_{1}}\right)} \frac{d t}{\vartheta_{1}}
\end{aligned}
$$

By doing some substitution and using equation [3.471,9] in [18], we can obtain the (10). In which, $K_{v}(\bullet)$ is the modified Bessel function of the second kind and $\mathrm{v}^{\text {th }}$ order.

Similarity we can formulate (11). In which, we denote:

$$
\vartheta_{2}=1-\gamma_{t h} A_{2}, \theta_{2}=\frac{C_{2}}{\vartheta_{2}}+\frac{\gamma_{t h} B_{2} D_{2}}{\vartheta_{2}^{2}}, \xi_{2}=E_{2}+\gamma_{t h} D_{2}\left(\frac{C_{2}}{\vartheta_{2}}+\frac{\gamma_{t h} B_{2} D_{2}}{\vartheta_{2}^{2}}\right)
$$

\subsection{Asymptotic analysis}

In this section, the asymptotic outage probability at high SNRs is proposed and investigated. In this case, we assume that $P_{1}=P_{2}=\varepsilon P_{r}(\varepsilon>0)$ (without significant loss of generality). Then we have:

$$
\beta_{\infty}=\frac{r}{y_{r}}=\sqrt{\frac{P_{r}}{\varphi_{1} P_{1}+\varphi_{1} P_{1} \kappa_{1}^{2}+\varphi_{2} P_{2}+\varphi_{2} P_{2} \kappa_{2}^{2}+\sigma_{r}^{2}}}=\frac{1}{\sqrt{\varepsilon \varphi_{1}\left(1+\kappa_{1}^{2}\right)+\varepsilon \varphi_{2}\left(1+\kappa_{2}^{2}\right)}}
$$

Moreover, the SNR in (7) and (8) can be rewritten as (25) and (26), respectively. 


$$
\begin{aligned}
& \gamma_{1}^{\infty}=\frac{\varphi_{2}}{A_{1} \varphi_{2}+B \varphi_{1}} \\
& \gamma_{2}^{\infty}=\frac{\varphi_{1}}{A_{2} \varphi_{1}+C \varphi_{2}}
\end{aligned}
$$

Where we denote $A_{1}=\kappa_{2}^{2}+\kappa_{r}^{2}\left(1+\kappa_{2}^{2}\right), B=\kappa_{1}^{2}+\kappa_{r}^{2}\left(1+\kappa_{1}^{2}\right) \frac{\sigma_{2}^{2}}{\sigma_{1}^{2}}, A_{2}=\kappa_{1}^{2}+\kappa_{r}^{2}\left(1+\kappa_{1}^{2}\right)$, and $C=\kappa_{2}^{2}+\kappa_{r}^{2}\left(1+\kappa_{2}^{2}\right) \frac{\sigma_{1}^{2}}{\sigma_{2}^{2}}$.

Theorem 2. The asymptotic expression of the outage probability $P_{\text {out_l }}$ and $P_{\text {out_2 }}$ at the source nodes $S 1, S 2$ of the proposed system could be calculated by the following:

$$
\begin{aligned}
& P_{\text {out_1 }}^{\infty}=1-a^{2} \sum_{l=0}^{\infty} \sum_{k=0}^{\infty} \sum_{m=0}^{l} \frac{K^{l+k} b^{m+k-1}(m+k) !}{l ! m !(k !)^{2}}\left(\frac{\gamma_{t h} B}{1-\gamma_{t h} A_{1}}\right)^{m} \times\left[\frac{b \gamma_{t h} B}{1-\gamma_{t h} A_{1}}+b\right]^{-m-k-1} \\
& P_{\text {out_- }}^{\infty}=1-a^{2} \sum_{l=0}^{\infty} \sum_{k=0}^{\infty} \sum_{m=0}^{l} \frac{K^{l+k} b^{m+k-1}(m+k) !}{l ! m !(k !)^{2}}\left(\frac{\gamma_{t h} C}{1-\gamma_{t h} A_{2}}\right)^{m} \times\left[\frac{b \gamma_{t h} C}{1-\gamma_{t h} A_{2}}+b\right]^{-m-k-1}
\end{aligned}
$$

\section{Proof:}

The asymptotic outage probability of the source node S1 can be formulated by the followings:

$$
\begin{aligned}
& P_{\text {out } \_1}^{\infty}=F_{\gamma_{1}^{\infty}}\left(\gamma_{t h}\right)=\operatorname{Pr}\left(\gamma_{1}^{\infty}<\gamma_{t h}\right)=\operatorname{Pr}\left(\frac{\varphi_{2}}{A_{1} \varphi_{2}+B \varphi_{1}}<\gamma_{t h}\right) \\
& P_{\text {out_1 }}^{\infty}=\operatorname{Pr}\left[\varphi_{2}\left(1-\gamma_{t h} A_{1}\right)<\gamma_{t h} B \varphi_{1}\right]=\operatorname{Pr}\left(\varphi_{2}<\frac{\gamma_{t h} B \varphi_{1}}{1-\gamma_{t h} A_{1}}\right)
\end{aligned}
$$

Furthermore

$$
P_{\text {out }-1}^{\infty}=\int_{0}^{\infty} F_{\varphi_{2}}\left(\frac{\gamma_{t h} B \varphi_{1}}{1-\gamma_{t h} A_{1}}\right) f_{\varphi_{1}}\left(\varphi_{1}\right) d \varphi_{1}
$$

After that, we have:

$$
P_{\text {out } 1}^{\infty}=1-a^{2} \sum_{l=0}^{\infty} \sum_{k=0}^{\infty} \sum_{m=0}^{l} \frac{K^{l+k} b^{m+k-1}}{l ! m !(k !)^{2}}\left(\frac{\gamma_{t h} B}{1-\gamma_{t h} A_{1}}\right)^{m} \times \int_{0}^{\infty} \varphi_{1}^{m+k} \times e^{-b\left[\frac{\gamma_{t h} B \varphi_{1}}{1-\gamma_{t h} A_{1}}+\varphi_{1}\right]} d \varphi_{1}
$$

By using equation [3.351,3] in [18] we can obtain the (25). By the same way for the source node S2 we formulate the (26).

\section{RESULTS AND DISCUSSION}

In this section, some simulation results are proposed to investigate the system performances of the proposed network under hardware impairment over the Rician fading channels. Both in theoretical and Monte Carlo simulation results evaluate the system performance analysis. All system simulation parameters are presented in Table 1.

Table 1. Simulation parameters

\begin{tabular}{lll}
\hline Symbol & Name & Values \\
\hline$\lambda_{1}$ & Mean of $\left|g_{1}\right|^{2}$ & 1 \\
$\lambda_{2}$ & Mean of $\left|g_{2}\right|^{2}$ & 1 \\
$\mathrm{~K}$ & Rician K-factor & 3 \\
$\mathrm{P}_{1}=\mathrm{P}_{2}=\mathrm{P}_{\mathrm{r}}=\mathrm{P}$ & Source power to noise ratio & $0-40 \mathrm{~dB}$
\end{tabular}




\begin{tabular}{lll}
$\kappa_{1}=\kappa_{2}=\kappa_{r}=\kappa$ & Hardware impairment levels & $0-0.3$ \\
$\mathrm{R}$ & Source rate & $1 \mathrm{bit} / \mathrm{s} / \mathrm{Hz}$ \\
\hline
\end{tabular}

In this analysis, Figure $3 \mathrm{a}$ and $3 \mathrm{~b}$ show the influence of $\mathrm{K}$ on the outage probability and throughput of the model system, respectively. In this simulation process, the main parameters of the proposed system are set as the following: $\mathrm{P}=20 \mathrm{~dB}, \kappa_{1}=\kappa_{2}=\kappa_{\mathrm{r}}=0 ; 0.15 ; 0.25$. Figure 3 shows that the outage probability decreased and the throughput increased crucially while $\mathrm{K}$ varied from 0 to 8 . Moreover, the analytical results agree well with the Monte Carlo simulation results.

On another hand, Figure $4 \mathrm{a}$ and $4 \mathrm{~b}$ illustrate the effect of $\kappa$ on the outage probability and the achievable throughput of the model system. Here, $\mathrm{P}_{1}=\mathrm{P}_{2}=\mathrm{P}_{\mathrm{r}}=\mathrm{P}$ is set at $15 ; 20 ; 25 \mathrm{~dB}$. From the simulation, it is clear fond that the achievable throughput increases and the outage probability decrease significantly while $\kappa_{1}=\kappa_{2}=\kappa_{\mathrm{r}}$ varies from 0 to 0.3 . In these cases, the figures reveal that the simulation results match tightly with analytical expressions in section 3 .

Moreover, Figure $5 \mathrm{a}$ and $5 \mathrm{~b}$ present the effect of the $\mathrm{P}$ on the outage probability and the achievable throughput with $\kappa_{1}=\kappa_{2}=\kappa_{\mathrm{t}}=0.1 ; 0.2$ for the proposed system. From the results, it is shown that the achievable throughput increases and the outage probability decreased significantly when the $\mathrm{P}$ increased from 0 to 40 $\mathrm{dB}$. In these figures, all the analytical and the simulation results are the same values.

In the same way, the influence of the system rate $\mathrm{R}$ on the outage probability and the achievable throughput of the system model with $\mathrm{P}=20 \mathrm{~dB}$ and $\kappa_{1}=\kappa_{2}=\kappa_{\mathrm{t}}=0.025 ; 0.05$ are illustrated in the Figure $6 \mathrm{a}$ and $6 \mathrm{~b}$. Figure $6 \mathrm{a}$ showed that the outage probability increases crucially with increasing the rate R. However, the system throughput only increased in the interval $\mathrm{R}$ from 0 to the optimal value around 2. After that, it significantly fell to 0 at the rate around 4 . In particular, the simulation lines wholly matched with the analytical lines in all the above figures.

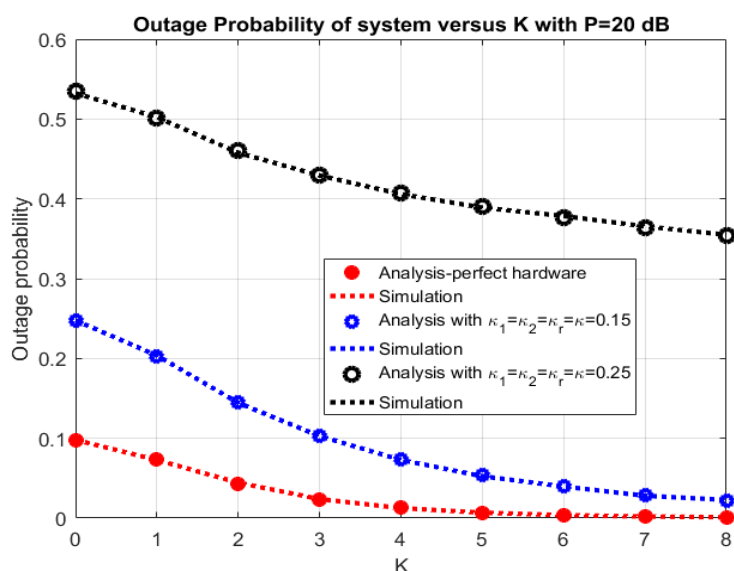

(a)

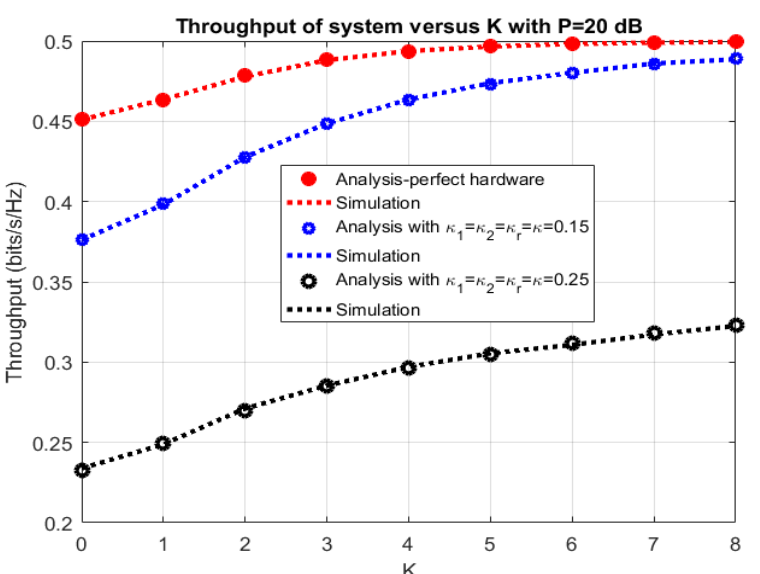

(b)

Figure 3. Outage probability (a) and achievable throughput (b) of the system model versus $\kappa$ 


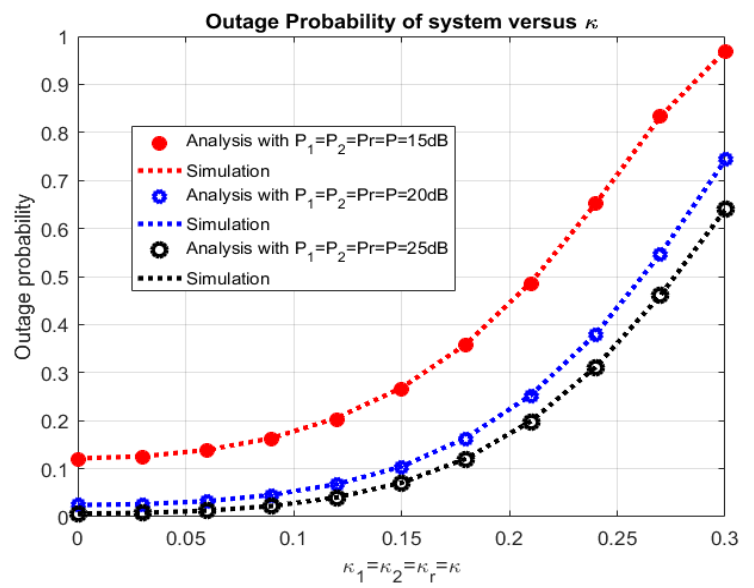

(a)

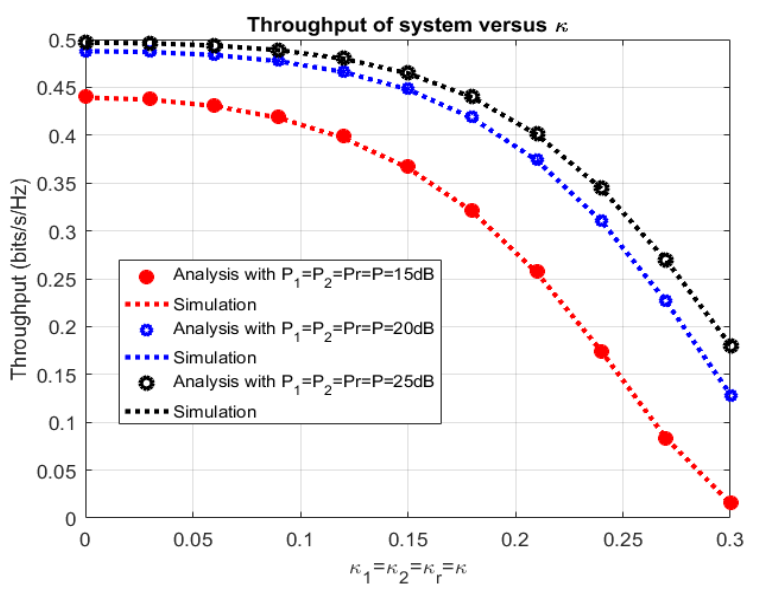

(b)

Figure 4. Outage probability (a) and achievable throughput (b) of the system model versus $\kappa$

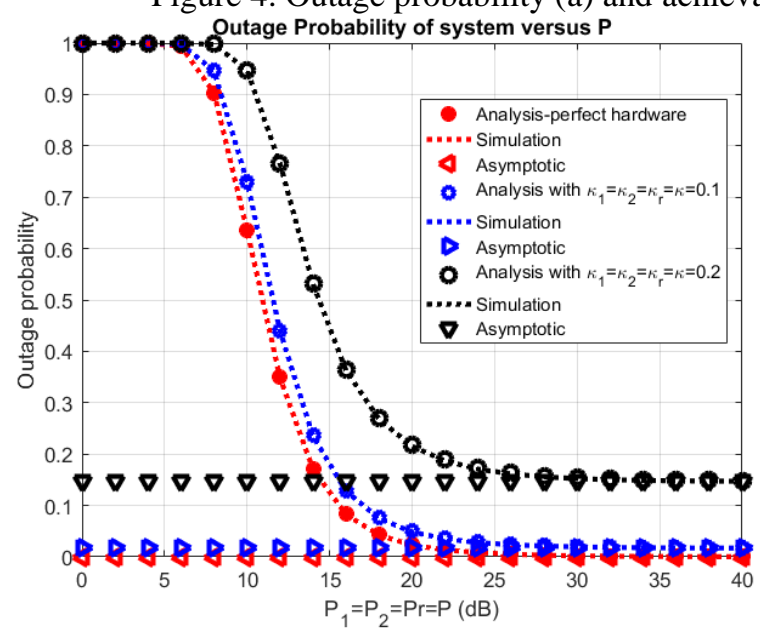

(a)

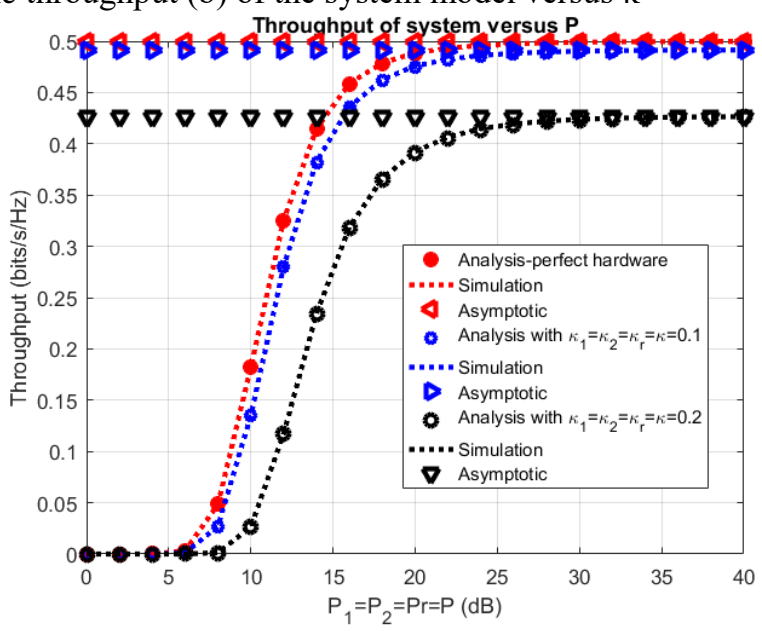

(b)

Figure 5. Outage probability (a) and achievable throughput (b) of the system mode versus P

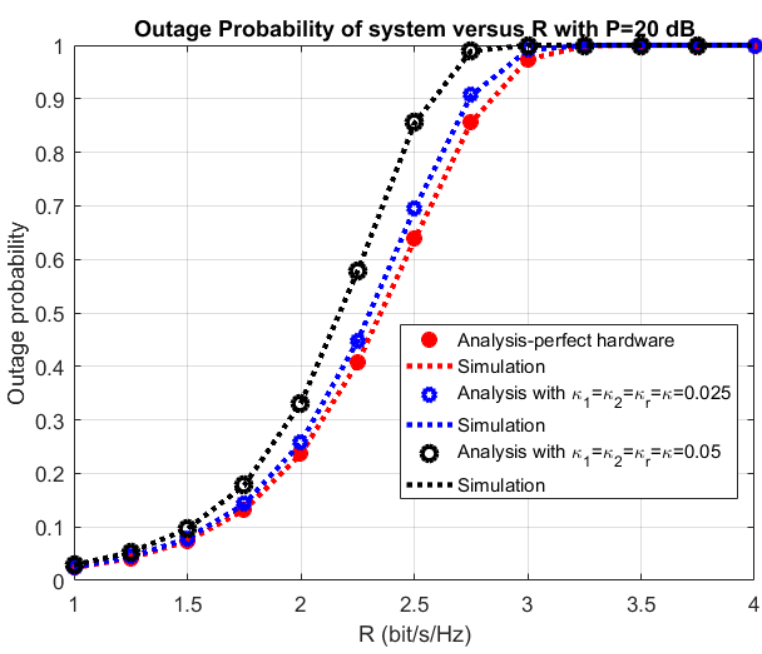

(a)

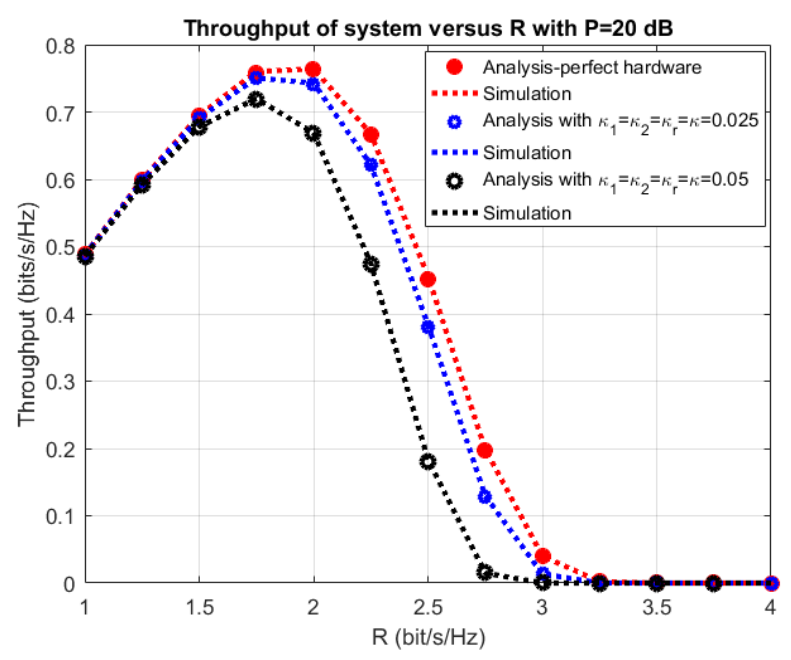

(b)

Figure 6. Outage probability (a) and achievable throughput (b) of the system mode versus R 


\section{CONCLUSION}

In this paper, we investigate the system performance of the two-way half-duplex relay network under hardware impairment condition. The analytical expressions of the outage probability and achievable throughput with the exact closed form and asymptotic form were proposed and derived. Furthermore, the analytical results are also demonstrated and convinced by Monte-Carlo simulation, and the analytical and the simulation results are matched well with each other for all possible system parameters. The research results can provide the essential recommendations for the communication network research and practice directions.

\section{REFERENCES}

[1] Bi S., Ho C. K., Zhang R., "Wireless powered communication: Opportunities and challenges," IEEE Communications Magazine, vol. 53, no. 4, pp. 117-125, Apr 2015.

[2] Chen Xiaoming, Derrick Wing Kwan Ng and Hsiao-Hwa Chen, "Secrecy Wireless Information and Power Transfer: Challenges and Opportunities," IEEE Wireless Communications, vol. 23, no. 2, pp. 54-61, Apr 2016.

[3] Niyato D., Kim D. I., Maso M., Han Z., "Wireless Powered Communication Networks: Research Directions and Technological Approaches," IEEE Wireless Communications, vol. 24, no. 6, pp. 88-97, 2017.

[4] Salem A., Hamdi K. A., "Wireless Power Transfer in Multi-Pair Two-Way AF Relaying Networks," IEEE Transactions on Communications, vol. 64, no. 11, pp. 4578-4591, Nov. 2016.

[5] Silva S., Ardakani M., Tellambura C., "Relay Selection for Cognitive Massive MIMO Two-Way Relay Networks," 2017 IEEE Wireless Communications and Networking Conference (WCNC), 2017, 03.

[6] Amarasuriya G., Tellambura C., Ardakani M., "Multi-Way MIMO Amplify-and-Forward Relay Networks with Zero-Forcing Transmission," IEEE Transactions on Communications, vol. 61, no. 12, pp. 4847-4863, Dec 2013.

[7] Peng M., Yang C., Zhao Z., Wang W., Chen, H., "Cooperative network coding in relay-based IMT-advanced systems," IEEE Communications Magazine, vol. 50, no. 4, pp. 76-84, Apr 2012.

[8] Yi Z., Ju M., Kim, I., "Outage Probability and Optimum Power Allocation for Analog Network Coding," IEEE Transactions on Wireless Communications, vol. 10, no. 2, pp. 407-412. Feb 2011.

[9] Upadhyay P. K., Prakriya S., "Performance of Analog Network Coding with Asymmetric Traffic Requirements," IEEE Communications Letters, vol. 15, no. 6, pp. 647-649, Jun 2011.

[10] Mehemed A., Hamouda W., "Asymptotic Outage Probability for Amplify-and-Forward CDMA Systems over Nakagami-m Fading Channels," 2012 IEEE Vehicular Technology Conference (VTC Fall), Sep 2012.

[11] Yang J., Fan P., Duong T. Q., Lei X., "Exact Performance of Two-Way AF Relaying in Nakagami-m Fading Environment," IEEE Transactions on Wireless Communications, vol. 10, no. 3, pp. 980-987. Mar 2011.

[12] Hwang K., Ju M., Alouini M., "Outage Performance of Opportunistic Two-Way Amplify-and-Forward Relaying with Outdated Channel State Information," IEEE Transactions on Communications, vol. 61, no. 9, pp. 3635-3643, Sep 2013.

[13] Upadhyay P. K., Yadav S., "On the Performance of Cellular Two-Way Relay Systems with Analog Network Coding and Multiuser Diversity," Wireless Personal Communications, vol. 72, no. 4, pp. 2705-2720, Apr 2013.

[14] Atapattu S., Jing Y., Jiang H., Tellambura C., "Relay Selection Schemes and Performance Analysis Approximations for Two-Way Networks," IEEE Transactions on Communications, vol. 61, no. 3, pp. 987-998, Mar 2013.

[15] Tan N. Nguyen, Phuong T. Tran, T. H. Q. Minh and Miroslav Voznak, "Two-Way Half Duplex Decode and Forward Relaying Network with Hardware Impairment over Rician Fading Channel: System Performance Analysis," Elektronika Ir Elektrotechnika, vol. 24, no. 2, pp. 74-78, Apr 2018.

[16] Tan N. Nguyen, T. H. Q. Minh, Phuong T. Tran and Miroslav Voznak, "Adaptive Energy Harvesting Relaying Protocol for Two-Way Half Duplex System Network over Rician Fading Channels," Wireless Communications and Mobile Computing, vol. 2018, pp. 1-10, Apr 2018.

[17] Suraweera H., Karagiannidis G., Smith P., "Performance analysis of the dual-hop asymmetric fading channel," IEEE Transactions on Wireless Communications, vol. 8, no. 6, pp. 2783-2788, Jun 2009.

[18] Gradshteĭn I. S., I. M. Ryzhik and Alan Jeffrey, Table of Integrals, Series, and Products, Academic Press, 2000.

[19] Bhatnagar M. R., "On the Capacity of Decode-and-Forward Relaying over Rician Fading Channels," IEEE Communications Letters, vol. 17, pp. 6, pp. 1100-1103, Jun 2013. 